\title{
Omalizumab Has a Place in Therapy for Asthma Disease Management
}

\author{
Lawrence J. Pesko, MS, DSc, RPh
}

A sthma is a complex disease because its presentation can vary considerably from patient to patient. The nature of asthma often requires physicians to use a multifaceted approach to ensure optimal outcomes (i.e., control of symptoms) and to prevent asthma exacerbations and increased health care utilization. Evidence-based disease management guidelines have been developed and published to aid in the diagnosis of asthma and to help guide treatment decisions. In 2007, the National Asthma Education and Prevention Program (NAEPP) released its updated guidelines for the management and treatment of asthma. ${ }^{1}$ National guidelines such as these generally present the evidence currently available to support best practices for managing chronic diseases.

In the January 2008 issue of JMCP, Urbano reviewed 7 changes to the NAEPP guidelines that were considered by a small expert panel to be the most important and clinically relevant messages in the updated document. ${ }^{2}$ The bulk of these changes address diagnosis, successful comprehensive management, assessing disease severity, monitoring disease control, and managing asthma exacerbations. Some of the changes deal directly with medication use and choice. Because there is often a lag between the release of a new guideline and implementation in clinical practice, there is an opportunity to provide education and develop strategies that incorporate new recommendations into current programs.

The management and treatment of asthma remain challenging for all health care stakeholders-patients, providers, and payers. Unlike other chronic diseases such as heart disease, which become increasingly prevalent with age, asthma affects patients across the age spectrum. Over the past 25 to 30 years, the prevalence of asthma has continued to grow at an alarming rate, with the greatest increase seen among children., ${ }^{3,4}$ Current estimates cite the overall prevalence of asthma in the United States at approximately 22 million people, or about $7.7 \%$ of the population. ${ }^{5}$

Despite growing recognition of asthma as a chronic disease and strides toward improving its treatment, the financial burden of this disease is enormous. The total direct and indirect costs associated with asthma are approximately $\$ 20$ billion annually, ${ }^{6}$ the greatest proportion of which is attributable to poorly controlled asthma. ${ }^{7}$ According to The Epidemiology and Natural History of Asthma: Outcomes and Treatment Regimens

Note: This article is the subject of an editorial that appears on pages 289-293 of this issue.
(TENOR) study, a prospective, observational, multicenter, 3-year U.S. study of 4,756 patients with severe or difficult-totreat asthma, the highest percentage of health care utilization in the prior 3 months was linked to patients with severe asthma. ${ }^{8}$

The mandate for improving the care of asthma is clear. However, the existence of guidelines does not always ensure improved patient care. In a 2-year retrospective study of claims data from more than 4 million United Healthcare enrollees nationwide, overall adherence to evidence-based practice guidelines was 59\% for select chronic conditions, including coronary artery disease, congestive heart failure, hyperlipidemia, asthma, and diabetes. ${ }^{9}$ For adults with persistent asthma, $79 \%$ of the commercially insured population filled a prescription for inhaled corticosteroid (ICS) therapy, whereas only 58\% of Medicare enrollees filled an ICS prescription. ${ }^{9}$ Adherence to national asthma guidelines among inner-city physicians is even poorer. In a survey of 202 primary care providers in East Harlem, New York, $62 \%$ reported adhering to the National Heart, Lung and Blood Institute (NHLBI) asthma guidelines for ICS use. ${ }^{10}$ However, 34\% reported adherence to the NHLBI guidelines recommendations for peak flow monitoring, $10 \%$ for referrals to allergy testing, and $9 \%$ for using an asthma action plan. It was also noted that greater provider self-efficacy was associated with greater adherence to peak flow monitoring, ICS use, and use of an asthma action plan. ${ }^{10}$

Even with various expert-derived guidelines that provide asthma treatment strategies, many patients remain symptomatic and their asthma symptoms suboptimally controlled.,11,12 In the retrospective study by Thier et al., patient adherence to ICS therapy was poor, even though $78 \%$ of all adults with persistent asthma filled an ICS prescription. Among this population, 37\% were nonadherent to ICS therapy. In children with persistent asthma, nonadherence to ICS medications was $42 \% .{ }^{9}$ A retrospective cross-sectional study of nearly 12 million elderly adults showed that physicians were not adherent to asthma therapy recommendations from the NAEPP Expert Panel Report (EPR)-2 guidelines. This analysis, based on data from the National Ambulatory Medical Care Survey, found that individuals aged 65 years or older were $54 \%$ as likely to be prescribed controller medications compared with those aged 35 to 64 years. Those aged 65 years or older were also 48\% less likely to be prescribed long-acting beta- 2 agonists (LABAs), $49 \%$ less likely to be prescribed short-acting beta agonists (SABAs), and $49 \%$ less likely to be prescribed combination ICS and LABA 
therapy. ${ }^{13}$ These data point to the fact that physician and patient adherence to clinical practice guidelines and recommended therapies are often suboptimal and may be contributing to poor outcomes and increased health care utilization.

Medication management-balancing efficacy, safety, and pharmacoeconomic data-is a central component of managed care. As disease state information evolves, managed care medical and pharmacy directors must keep abreast of new information and monitor formulary management options to determine if the currently employed strategies for medication management reflect current practice guidelines. This task may be especially important for diseases that affect large numbers of individuals and are associated with significant negative economic consequences if not managed effectively.

The release of updated clinical guidelines provides an opportunity for managed care organizations (MCOs) to reassess their disease management programs. Continuous monitoring is needed to evaluate the outcomes achieved by these programs so they can be adjusted, as necessary, to optimize the overall quality of care. The inclusion of omalizumab (Xolair) in the NAEPP guidelines offers another treatment option for patients who have immunoglobulin E (IgE)-mediated asthma. Health plans, however, need to identify, through pharmacy and medical data claims, the prevalence of uncontrolled asthma in their populations and then reassess their formulary management strategies to target the appropriate use of omalizumab in select populations.

\section{Identification of "At-Risk" Populations}

Implementing strategies to identify high risk patients-those with difficult-to-treat or uncontrolled moderate-to-severe asthma-is in the best interest of MCOs. From a disease standpoint, it appears that the costs associated with moderate-to-severe asthma and the resulting morbidity and mortality are substantial. These data imply that identifying patients with poorly controlled asthma and employing implementation strategies that provide ongoing education, as well as therapeutic alternatives, to improve control are important cost-management strategies for MCOs.

Measures have been evaluated to identify at-risk patients in large patient populations. Pharmacy-driven measures that include the frequency of dispensing events for SABAs or oral corticosteroids can predict future emergency care. ${ }^{14}$ In a retrospective review of patients with persistent asthma in a managed care population, use of 5 to 13 canisters of a SABA in the first year was associated with an increased risk of emergency hospital utilization in the second year compared with use of only 0 to 4 canisters in the first year (odds ratio $[\mathrm{OR}]=2.2,95 \% \mathrm{CI}=1.2-3.8$ ). Similarly, use of more than 2 courses of oral corticosteroids in the first year was associated with an increased risk of emergency hospital utilization in the second year compared with use of 0 to 2 courses of oral corticosteroids (OR=2.6, 95\% CI = 1.5-4.5)..$^{14}$

In addition to clinical measures to determine asthma severity and control, the NAEPP guidelines suggest specific quality- of-life (QOL) measures, such as the Asthma Quality of Life Questionnaire (AQLQ) and the Asthma Control Test (ACT), to assess the impact of asthma on patient QOL and daily functioning. ${ }^{1}$ AQLQ scores were an adequate measure of control, as determined by Bateman et al..$^{15}$ This study showed that, across a range of asthma disease severity, well-controlled asthma patients had consistently higher AQLQ scores at end point and larger AQLQ improvements from baseline than patients who were not well controlled. This correlation suggests a relationship between guideline-based asthma control and improvement in QOL. ${ }^{15}$

Similarly, the ACT has been shown to be a practical and simple tool that can effectively assess the level of asthma control, with or without lung function testing. ${ }^{16}$ This brief 5 -item questionnaire measures several different areas of asthma control, including symptoms, rescue inhaler usage, and the impact of asthma on everyday functioning. It is important to note, however, that the 2007 NAEPP guidelines state that lack of experience with QOL surveys and the time needed to administer these instruments preclude their general adoption for routine encounters. ${ }^{1}$ As more information becomes available, the use of QOL measures may become more commonplace. Questionnaires have been used, in some cases in addition to administrative data, to identify patients at highest risk for emergency care and may offer MCOs another means to identify at-risk patients. ${ }^{17-19}$

The NAEPP 2007 update also addresses the role of spirometry in the evaluation of asthma control. It is recommended that the forced expiratory volume in 6 seconds $\left(\mathrm{FEV}_{6}\right)$, forced vital capacity $(\mathrm{FVC})$ and the $\mathrm{FEV}_{1} / \mathrm{FVC}$ ratio be done before and following the use of a short-acting bronchodilator for the initial diagnosis of asthma and should be subsequently used to assess the risk for future adverse events. ${ }^{1}$ While these measures are useful in the physician office, they are generally not available in administrative databases to help MCOs identify members who are at risk for future adverse events secondary to asthma so that interventions to reduce risk can be initiated. Moreover, assessments of lung function alone may not provide a clear picture of asthma control without additional information about symptoms and their effect on a patient's QOL. ${ }^{11,20}$ Even with these additional measures for assessing control, accurate assessment can be difficult.

\section{The Case for Omalizumab in Medication Management}

The updated NAEPP guidelines (2007) include an expansion of the stepwise approach for treating asthma. Unlike previous NAEPP guidelines, which had 4 steps based on asthma severity, the current edition now uses 6 steps to better address those whose asthma remains uncontrolled with traditional therapies. ${ }^{1}$ According to the stepwise approach as outlined in the current update and previous (2002) NAEPP guidelines, allergen immunotherapy should be considered in Steps 2-4, regardless of age. The guidelines recommend that immunotherapy should be considered in patients whose symptoms occur year round or during a significant portion of the year and are difficult to 
control pharmacologically. ${ }^{1}$

As noted by Urbano et al., ${ }^{2}$ one significant change in the NAEPP guidelines is the inclusion of omalizumab as adjunctive therapy for youths aged 12 years or older and adults with perennial allergies and severe persistent asthma that is not adequately controlled with the combination of ICS and a LABA. Omalizumab, a recombinant deoxyribonucleic acid (DNA)derived human monoclonal antibody, is approved by the U.S. Food and Drug Administration (FDA) for use in patients with moderate-to-severe persistent asthma. ${ }^{21,22}$ Omalizumab binds to IgE, forming complexes that inhibit the response to allergens. ${ }^{21,22}$ Sources of relevant perennial allergens include dust mites, cockroaches, cats, and dogs.

The NAEPP 2007 update reserves the use of omalizumab for Steps 5 and 6 which, by definition, are adjunctive therapy for patients aged 12 years or older who have allergies with severe persistent asthma. In such cases-patients who remain uncontrolled despite traditional therapies, especially those with IgE-mediated allergic asthma-adjunctive therapy with agents that target the underlying allergic component may be beneficial. Identification of the patient populations that could most benefit from omalizumab therapy remains a challenge, however. According to package labeling, omalizumab is "indicated for adults and adolescents (12 years of age and above) with moderate to severe persistent asthma who have a positive skin test or in vitro reactivity to a perennial aeroallergen and whose symptoms are inadequately controlled with inhaled corticosteroids."22

The clinical benefits of omalizumab as adjunctive therapy have been detailed in the literature. Clinical studies have found that combining omalizumab with ICS reduces asthma exacerbations, ${ }^{23,24}$ improves lung function, ${ }^{23,24}$ and results in greater reductions in steroid dosing compared with placebo. ${ }^{23-25}$ Improvements in QOL have also been reported in patients receiving omalizumab compared with placebo. ${ }^{25,26}$ Unlike other adjunctive therapies that also improve outcomes and reduce steroid dosing, omalizumab has demonstrated added benefit when combined with high-dose ICS and LABA in the management of severe persistent allergic asthma. ${ }^{26}$

The National Institute for Health and Clinical Excellence (NICE) noted in its appraisal (2007) that omalizumab may be considered as add-on therapy only to optimized, standard therapy in adults and adolescents aged 12 years or older who have severe, unstable disease. ${ }^{27}$ Use of and compliance with the standard treatments, including high-dose ICS therapy and LABAs in addition to leukotriene receptor agonists, theophylline, oral corticosteroids, and oral beta-2 agonists, are recommended before considering omalizumab as add-on therapy. In order to be considered for omalizumab therapy, the NICE guidelines define the criteria that patients must meet to establish the existence of severe, unstable allergic asthma: (a) confirmation of IgE-mediated allergic asthma by clinical history and allergy skin test; and (b) at least 2 severe exacerbations requiring hospitalization within the previous year, or at least 3 severe exacerbations within the previous year, with 1 requiring hospitalization or 2 requiring treatment or monitoring beyond the patient's usual regimen. ${ }^{27}$ The NICE guidelines also recommend discontinuation of omalizumab after 16 weeks if there is no adequate response to therapy based on a full clinical assessment that includes degree of asthma control, QOL, control of exacerbations, avoidance of unscheduled health care utilization, spirometry and peak expiratory flow measures, and physician global assessment of treatment efficacy. ${ }^{27}$

The NICE appraisal of omalizumab included evaluation of the results from the Investigation of Omalizumab in Severe Asthma Treatment (INNOVATE), a randomized, double-blind, multicenter study in patients with poorly controlled severe persistent asthma that measured clinically significant exacerbations and clinically significant severe exacerbations in patients receiving omalizumab as add-on therapy. The NICE committee noted that the rate of clinically significant exacerbations (the primary efficacy outcome) in the primary intention-to-treat population in INNOVATE was 0.74 for the omalizumab group versus 0.92 for the placebo group (rate ratio $=0.806,95 \% \mathrm{CI}=0.600-1.083$, $P=0.153$ ), which was not statistically significant until after posthoc adjustments were made. Other INNOVATE results included statistically significant reductions in clinically significant severe asthma exacerbations and greater improvement in AQLQ scores for omalizumab compared with placebo. ${ }^{27}$

In addition to the post hoc adjustments, NICE criticized the INNOVATE study on several points such as the exclusion of $13 \%$ of randomized patients prior to the implementation of the protocol amendments. The NICE committee wrote, "There were aspects of the INNOVATE RCT [randomized controlled trial] that led to uncertainty, including lack of detail on concealment of treatment allocation, the possibility of inadequate double blinding, selection bias, and exclusion of randomised patients for the intention-to-treat population." ${ }^{27}$ These uncertainties led NICE to question "the improvement observed for the primary efficacy outcome." ${ }^{27}$ Following testimony from both clinical specialists and patient experts, NICE concluded that "omalizumab as an add-on to optimised standard therapy is more clinically effective in particular groups of patients than optimised standard therapy alone."27

In clinical studies, omalizumab has been shown to reduce asthma-related hospitalizations and emergency room visits. ${ }^{26,28}$ Ayres et al. compared the safety and efficacy of omalizumab versus best standard care in patients with moderate-to-severe asthma in a randomized, open-label, multicenter study. ${ }^{28}$ The primary efficacy measure (annualized asthma deterioration-related incidents defined as asthma-related need for systemic antibiotics or corticosteroids, missed school or work, unscheduled physician office visits or hospitalization or emergency room visits) was 9.76 with best standard care alone $(n=106)$ versus $4.92(n=206)$ per patient-year with omalizumab $(P<0.001){ }^{28}$ In the INNOVATE study, the addition of omalizumab to high-dose ICS and LABA 
during the 28-week study period resulted in fewer total emergency visits compared with placebo added to high-dose ICS and LABA (50 vs. 93, $P=0.038$ ). The omalizumab group also had nonsignificantly lower rates of hospital admissions (13 vs. 25 for placebo, $P=0.117$ ) and unscheduled doctor visits ( 28 vs. 54 for placebo, $P=0.090) .{ }^{26}$

Administration of omalizumab is not without risks. ${ }^{1}$ Pain and bruising can occur in 5\%-20\% of patients following administration and malignant neoplasms were reported in $0.5 \%$ of patients compared with $0.2 \%$ in those treated with placebo. Anaphylaxis has also been reported in $0.2 \%$ of patients. Because of the risk of anaphylaxis, clinicians that decide to administer omalizumab must be prepared and equipped to identify and treat anaphylaxis should it occur. ${ }^{1}$ Additionally, the manufacturers of omalizumab added a black-box warning in 2007 to highlight the possibility of anaphylaxis, which includes bronchospasm, hypotension, syncope, urticaria, and/or angioedema of the throat or tongue..$^{22}$ The warning also notes that anaphylaxis has been reported from the time of the first dose and beyond 1 year of treatment. ${ }^{22}$ Physicians are also advised to be prepared to manage life-threatening anaphylaxis if it occurs, inform patients of the signs/symptoms of anaphylaxis, and instruct patients to seek medical care if any symptoms occur. ${ }^{22}$ The NAEPP guidelines suggest referral to an asthma specialist for consultation or comanagement if the patient is not well controlled, if the patient requires Step 4 therapy or higher, if immunotherapy or omalizumab are considered, or if the patient has had an exacerbation requiring a hospitalization. ${ }^{1}$

As with the identification of the at-risk patient with asthma, the optimal method of identifying the patient who is a candidate for omalizumab is complicated. Belliveau et al. noted in their review in JMCP (2005) that appropriate screening is needed to determine which patients would respond most favorably to omalizumab treatment, and that when this subset of patients is targeted, the reduction of medical resources could potentially offset the drug's high acquisition cost. ${ }^{29}$ In an accompanying editorial, Curtiss commented that based on the evidence to date, the efficacy of omalizumab beyond 7 months is unknown and the key to cost-effective use lies in selective and specific use. ${ }^{30}$

Currently, most health plans have placed prior authorization (PA) restrictions on omalizumab due to its high cost, the availability of other agents, and concerns regarding its safety, including the potential for anaphylaxis and lack of safety data beyond 1 year of treatment.

Since its approval by the FDA in 2003, the safety and efficacy of omalizumab and its appropriate use in asthma patients have been debated in the clinical literature. The release of the 2007 NAEPP update provides an opportunity to re-evaluate the place in therapy of omalizumab. At this point, the definition of the ideal candidate most likely to respond favorably to omalizumab therapy is not clear. Continued monitoring of outcomes for patients who receive omalizumab, however, will be essential and perhaps, with continued use, a pattern will evolve that better defines those who best respond to this therapy.

\section{Conclusion}

As noted previously by Urbano, ${ }^{2}$ currently available clinical guidelines serve as an ideal starting point for improving the quality of care for the asthma disease state. The NAEPP guideline update in August 2007 should help clinicians in assessing and monitoring asthma. With a shift in focus to measuring control with a combination of outcomes tools, the identification and treatment of patients with poorly controlled asthma may be improved. The integration of various outcomes measures will be required and should include clinical assessments, physician and patient-reported measures of productivity/avoidance of activity, and burden to society and the workplace. In certain high-risk patients with severe persistent asthma, omalizumab may be considered as a treatment option. The ideal candidate for omalizumab therapy is as of yet unknown. Therefore, continued evaluation of this treatment is needed to refine the parameters for targeting patients who may benefit most from omalizumab.

\section{Author}

LAWRENCE J. PESKO, MS, DSc, RPh, is Director of Pharmacy, Presbyterian Medical Services, Santa Fe, New Mexico.

AUTHOR CORRESPONDENCE: Lawrence J. Pesko, MS, DSc, RPh, Director of Pharmacy, Presbyterian Medical Services, 1422 Paseo de Peralta, P.O. Box 2267, Santa Fe, NM 87504-2267. Tel.: 505.820.3490; E-mail: larry_pesko@pmsnet.org.

\section{DISCLOSURES}

This commentary was sponsored by Genentech, Inc., manufacturer of omalizumab. The author reports no financial interest or other potential conflict of interest.

The author acknowledges Linda Wagner, Charlotte Kenreigh, and Dennis Bloshuk, editor, Strategic Healthcare Alliance, for their assistance in the preparation and revision of this manuscript.

\section{REFERENCES}

1. National Heart, Lung, and Blood Institute, National Asthma Education and Prevention Program. Expert Panel Report 3. Guidelines for the Diagnosis and Management of Asthma. Bethesda, MD: National Institutes of Health; August 2007. NIH Publication No. 07-4051. Available at: http://www.nhlbi. nih.gov/guidelines/asthma/asthgdln.pdf. Accessed November 6, 2008

2. Urbano F, for a panel of 9 asthma disease specialists. Review of the NAEPP 2007 expert panel report-3 (EPR-3) on asthma diagnosis and treatment guidelines. J Manag Care Pharm. 2008;14:41-49. Available at: http:// www.amcp.org/data/jmcp/JMCPMaga_JanFeb\%2008_041-049.pdf.

3. Fireman P. Understanding asthma pathophysiology. Allergy Asthma Proc. 2003;24:79-83.

4. Fuhlbrigge AL, Adams RJ, Guilbert TW, et al. The burden of asthma in the United States: level and distribution are dependent on interpretation of the national asthma education and prevention program guidelines. Am J Respir Crit Care Med. 2002;166:1044-49. 
5. Akinbami L. Asthma prevalence, health care use, and mortality: United States 2003-05. November, 2006. National Center for Health Statistics, Centers for Disease Control and Prevention. Available at: http://www.cdc. gov/nchs/products/pubs/pubd/hestats/ashtma03-05/asthma03-05.htm. Accessed January 28, 2009.

6. American Lung Association, Epidemiology \& Statistics Unit, Research and Program Services. Trends in asthma morbidity and mortality. January 2009. Available at: http://www.lungusa.org/atf/cf/\%7B7a8d42c2-fcca-46048ade-7f5d5e762256\%7D/ASTHMA\%20JAN\%202009.PDF. Accessed January 28,2009 .

7. Sullivan SD, Rasouliyan L, Russo PA, Kamath T, Chipps BE; TENOR Study Group. Extent, patterns, and burden of uncontrolled asthma in severe and difficult-to-treat asthma. Allergy. 2007;62:126-33.

8. Dolan CM, Fraher KE, Bleecker ER, et al. Design and baseline characteristics of The Epidemiology and Natural History of Asthma: Outcomes and Treatment Regimens (TENOR) study: a large cohort of patients with severe or difficult-to-treat asthma. Ann Allergy Asthma Immunol. 2004;92:32-39.

9. Thier SL, Yu-Isenberg KS, Leas BF, et al. In chronic disease, nationwide data show poor adherence by patients to medication and by physicians to guidelines. Manag Care. 2008;17:48-52,55-57.

10. Wisnivesky JP, Lorenzo J, Lyn-Cook R, et al. Barriers to adherence to asthma management guidelines among inner-city primary care providers. Ann Allergy Asthma Immunol. 2008;101(3):264-70.

11. Bateman ED, Boushey HA, Bousquet J, et al.; GOAL investigators group. Can guideline-defined asthma control be achieved? The Gaining Optimal Asthma ControL study. Am J Respir Crit Care Med. 2004;170:836-44.

Available at: http://ajrccm.atsjournals.org/cgi/reprint/170/8/836. Accessed March 18, 2009.

12. Miller MK, Johnson C, Miller DP, Deniz Y, Bleeker ER, Wenzel SE; TENOR study group. Severity assessment in asthma: an evolving concept. $J$ Allergy Clin Immunol. 2005;116:990-95.

13. Navaratnam P, Jayawant SS, Pedersen CA, Balkrishnan R. Asthma pharmacotherapy prescribing in the ambulatory population of the United States: evidence of nonadherence to national guidelines and implications for elderly people. J Am Geriatr Soc. 2008;56:1312-17.

14. Schatz, M, Zeiger RS, Vollmer WM, et al. Development and validation of a medication intensity scale derived from computerized pharmacy data that predicts emergency hospital utilization for persistent asthma. Am J Manag Care. 2006;12:478-84

15. Bateman ED, Frith LF, Braunstein GL. Achieving guideline-based asthma control: does the patient benefit? Eur Respir J. 2002;20:588-95.

16. Nathan RA, Sorkness CA, Kosinski M, et al. Development of the asthma control test: a survey for assessing asthma control. J Allergy Clin Immunol. 2004;113:59-65.
17. Vollmer WM, Markson LE, O'Connor E, Frazier EA, Berger M, Buist AS. Association of asthma control with health care utilization. A prospective evaluation. Am J Respir Crit Care Med. 2002;165:195-99.

18. Peters D, Chen C, Markson LE, Allen-Ramey FC, Vollmer WM. Using an asthma control questionnaire and administrative data to predict health-care utilization. Chest. 2006;129:918-24.

19. Yurk RA, Diette GB, Skinner EA, et al. Predicting patient-reported asthma outcomes for adults in managed care. Am J Manag Care. 2004;10:321-28.

20. Luskin AT. Achieving asthma control: the need for risk assessment. Manag Care. 2005;14(Suppl 8):12-15.

21. Ruffin CG, Busch BE. Omalizumab: a recombinant humanized anti-IgE antibody for allergic asthma. Am J Health Syst Pharm. 2004;61:1449-59.

22. Genentech, Inc. Xolair (omalizumab) for subcutaneous use [prescribing information]. July, 2007.

23. Busse W, Corren J, Lanier BQ, et al. Omalizumab, anti-IgE recombinant humanized monoclonal antibody, for the treatment of severe allergic asthma. J Allergy Clin Immunol. 2001;108:184-90.

24. Solèr M, Matz J, Townley R, et al. The anti-IgE antibody omalizumab reduces exacerbations and steroid requirement in allergic asthmatics. Eur Respir J. 2001;18:254-61.

25. Holgate ST, Chuchalin AG, Hébert J, et al.; Omalizumab 011 International Study Group. Efficacy and safety of a recombinant anti-immunoglobulin E antibody (omalizumab) in severe allergic asthma. Clin Exp Allergy. 2004;34:632-38.

26. Humbert M, Beasley R, Ayres J, et al. Benefits of omalizumab as addon therapy in patients with severe persistent asthma who are inadequately controlled despite best available therapy (GINA 2002 step 4 treatment): INNOVATE. Allergy. 2005;60:309-16.

27. National Institute for Health and Clinical Excellence. Omalizumab for severe persistent allergic asthma. NICE technology appraisal guidance TA133, November 2007. Available at: www.nice.org.uk/nicemedia/pdf/ TA133Guidance.pdf. Accessed September 10, 2008.

28. Ayres JG, Higgins B, Chilvers ER, Ayre G, Blogg M, Fox H. Efficacy and tolerability of anti-immunoglobulin E therapy with omalizumab in patients with poorly controlled (moderate-to-severe) allergic asthma. Allergy. 2004;59:701-08

29. Belliveau PP, Lahoz MR. Evaluation of omalizumab from a health plan perspective. J Manag Care Pharm. 2005;11(9):735-45. Available at: http:// www.amcp.org/data/jmcp/formular_735-745.pdf.

30. Curtiss FR. Selectivity and specificity are the keys to cost-effective use of omalizumab for allergic asthma. J Manag Care Pharm. 2005;11(9):774-76. Available at: http://www.amcp.org/data/jmcp/editorial_774-776.pdf. 